Chirurg 2018 89:661-662

https://doi.org/10.1007/s00104-018-0676-4

(c) Springer Medizin Verlag $\mathrm{GmbH}$, ein Teil von Springer Nature 2018

CrossMark

Utz Settmacher - Silke Schüle

Klinik für Allgemein-, Viszeral- und Gefäßchirurgie, Universitätsklinikum Jena, Jena, Deutschland

\title{
Chirurgische Therapie peritonealer Malignome
}

tiven Therapie bei geeigneten Patienten. Daraus lässt sich die Hypothese ableiten, dass die HIPEC auch adjuvant oder prophylaktisch bei Risikopatienten zum Einsatz kommen könnte, um das Entstehen einer Peritonealkarzinose primär zu verhindern; dies wird derzeit in mehreren randomisierten Studien überprüft. Zum Einsatz der perioperativen Chemotherapie gibt es keine qualitativ hochwertigen Daten, sodass hierzu aus unserer Sicht keine allgemeine Empfehlung geäußert werden sollte. Unser eigenes Vorgehen beschreiben wir im Artikel von Schüle et al. wird das weitgehende Fehlen randomisierter Studien bzw. der Vergleich mit veralteten Chemotherapiepotokollen im Kontrollarm und die extreme Patientenselektion beanstandet. Andererseits ist die palliative systemische Chemotherapie am Peritoneum erwiesenermaßen nur schlecht wirksam, wodurch Patienten mit Peritonealkarzinose ein deutlich kürzeres medianes Überleben im Vergleich zu anderen Patienten im Stadium IV aufweisen.

Entscheidend für den langfristigen Therapieerfolg von CRS und HIPEC ist in der Tat eine sorgfältige Patientenselektion. Die besten Daten liegen heute zum kolorektalen Karzinom vor. Der in einer Vielzahl nichtrandomisierter Studien dargestellten Verbesserung des Gesamtüberlebens wurde durch die Aufnahme von CRS und HIPEC in die aktuellen S3Leitlinien für das kolorektale Karzinom inzwischen Rechnung getragen. Darüber hinaus konnte gezeigt werden, dass sowohl die Rezidiv- bzw. Überlebensraten als auch die operative Morbidität unmittelbar mit der peritonealen $\mathrm{Tu}$ morlast korrelieren. Dies unterstreicht die Bedeutung einer frühzeitigen opera-

\section{》) Bei geeigneten Patienten sollte die chirurgische Therapie so früh wie möglich erfolgen}

Magenkarzinome gehen besonders häufig synchron oder metachron mit einer Peritonealkarzinose einher. Da diese kaum in der üblichen bildgebenden Diagnostik zu detektieren ist, spielt die diagnostische Laparoskopie eine wichtige Rolle in der präoperativen Umfelddiagnostik. Auch für das Magenkarzinom gibt es Hinweise, dass bei geringer peritonealer Tumorlast, gemessen am Peritoneal Cancer Index (PCI), eine radikale chirurgische Therapie die Prognose verbessern kann, wobei diese in Analogie zu den lokal fortgeschrittenen Magenkarzinomen meist in ein perioperatives systemisches Chemotherapieprotokoll eingebettet wird. Einen Überblick über die aktuelle Datenlage beim peritoneal metastasierten Magenkarzinom gibt die

Beim Ovarialkarzinom stellt das Peritoneum den häufigsten Metastasierungsort und die häufigste Lokalisation von Arbeit von Brandl et al.
Tumorrezidiven dar. Hier stellt die maximale chirurgische Zytoreduktion (gefolgt von einer platinhaltigen systemischen Chemotherapie) den therapeutischen Standard dar. Im Gegensatz zum kolorektalen Karzinom und vor allem auch zum Magenkarzinom ist die Entscheidung zur Zytoreduktion („debulking“) weniger von der peritonealen Tumorlast (PCI) als von der technischen Machbarkeit abhängig. Die Vergleichbarkeit der Studienergebnisse ist allerdings durch eine sehr heterogene Definition des Begriffs „optimale Zytoreduktion“ eingeschränkt, wobei Tumorresiduen $>1 \mathrm{~cm}$ in jedem Fall vermieden werden sollten. In den USA wird die adjuvante intraperitoneale Chemotherapie als „early postoperative intraperitoneal chemotherapy " seit Jahren in vielen Zentren empfohlen, da mehrere Studien eine signifikante Verlängerung des Gesamtüberlebens nachweisen konnten. Insbesondere die Patientinnen mit peritonealen Tumorrezidiven und platinresistenten Tumoren haben kaum Therapiealternativen und können von einer lokalen Chemotherapie profitieren, wie von Horvath et al. dargestellt wird.

Die ausgedehnte Zytoreduktion mit großer Wundfläche, die häufig lange Operationsdauer und die durch das chirurgische Trauma ausgelöste systemische Inflammationsreaktion stellen besondere Anforderungen an die Narkoseführung während des Eingriffs sowie an das frühpostoperative Management auf der Intensivstation. Neben den hohen Flüssigkeitsverlusten und Volumenverschiebungen, für deren Ausgleich von Bleiler et al. der differenzierte Einsatz verschiedener Infusionslösungen empfohlen wird, betrifft dies auch die Folgen der Hyperthermie, die Nebenwirkungen 
\begin{tabular}{|l|l}
\hline & In eigener Sache
\end{tabular}

der applizierten Chemotherapie (Nephrotoxizität) und Elektrolytverschiebungen, die beispielsweise durch den Einsatz von Glukose als Trägerlösung für die intraperitoneale Chemotherapie ausgelöst werden können.

Die strenge Patientenselektion führt dazu, dass viele Patienten mit peritonealen Metastasen nicht für eine CRS und HIPEC infrage kommen. Vor allem Motilitätsstörungen des Dünndarms und Aszites können die Lebensqualität dieser Patienten auf ein Minimum reduzieren, sodass dringend Therapiealternativen erforderlich sind. Dies führte zur Entwicklung der „pressurized intraperitoneal aerosolic chemotherapy“ (PIPAC). Dabei wird über einen speziellen Vernebler die flüssige Chemotherapie in ein Aerosol überführt und auflaparoskopischem Wege ohne vorherige Zytoreduktion unter Druck in die Abdominalhöhle appliziert. Erste publizierte Ergebnisse und auch erste eigene Erfahrungen zeigen ein teils beeindruckendes Ansprechen bei geringer Morbidität und geringer Beeinträchtigung der Patienten durch den Eingriff. Auch aufgrund der minimal-invasiven Technik erscheint die PIPAC auf den ersten Blick als das modernere Verfahren und wird teilweise in eine Konkurrenzsituation zur „maximal-invasiven“ HIPEC gebracht. Die genauen Unterschiede von HIPEC und PIPAC hinsichtlich Indikationsstellung und Zielsetzung werden von Leebmann et al. beleuchtet.

\) PIPAC und HIPEC sollten nicht als konkurrierende, sondern als ergänzende Verfahren angesehen werden

Leider werden unserer Meinung nach weiterhin zu wenige Patienten mit malignen Erkrankungen des Peritoneums einer chirurgischen Therapie - sei sie nun kurativ intendiert oder „nur“ eine effektive Palliativmaßnahme - zugeführt. Mit diesem Themenheft möchten wir Ihnen zum einen den aktuellen Stand der Wissenschaft übermitteln, zum anderen aber auch gerne Ihre Aufmerksamkeit auf diese Erkrankung lenken, für die wir heute durchaus bessere Therapieoptio- nen als die reine systemische Chemotherapie haben. Wir wünschen Ihnen eine interessante und abwechslungsreiche Lektüre!
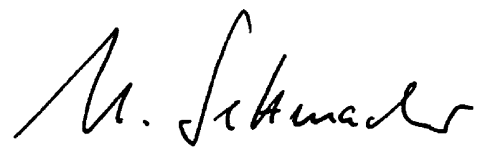

Prof. Dr. Utz Settmacher

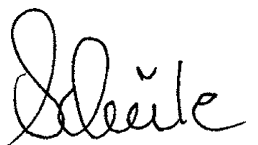

PD Dr. Silke Schüle

\section{Korrespondenzadresse}

Prof. Dr. U. Settmacher

Klinik für Allgemein-, Viszeral- und Gefäßchirurgie, Universitätsklinikum Jena Erlanger Allee 101, 07740 Jena, Deutschland utz.settmacher@med.uni-jena.de

Interessenkonflikt. U. Settmacher und S. Schüle geben an, dass kein Interessenkonflikt besteht.

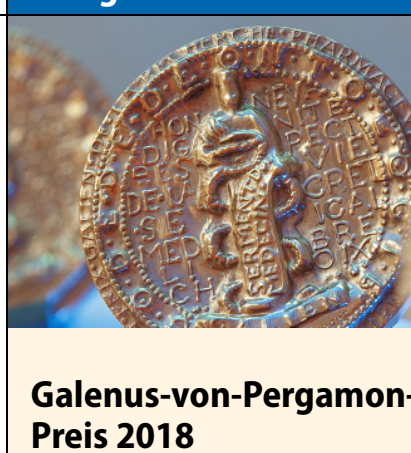

Mit dem von der Springer Medizin Verlag GmbH gestifteten Galenusvon-Pergamon-Preis werden herausragende Arzneimittelinnovationen gewürdigt. Über die Zuerkennung entscheidet eine unabhängige Expertenjury. Nominiert wurde:

\section{Zinplava ${ }^{\circledR}$ (Bezlotoxumab)}

Die Clostridium-difficile-Infektion (CDI) wird durch Toxine vermittelt, die das gastrointestinale Epithel schädigen und somit lokale Entzündungsreaktionen und eine starke Flüssigkeitssekretion verursachen. Problematisch ist, dass rund $20 \%$ der Patienten nach der Antibiotikatherapie der initialen CDI-Episode ein Rezidiv erleiden Bei etwa 30\% dieser Patienten kommt es mehrfach zur Rekurrenz. Dabei steigen mit zunehmender CDI-Rekurrenz auch die Morbidität und Mortalität.

Mit Bezlotoxumab (Zinplava ${ }^{\circledR}$ ) von MSD Sharp \& Dohme steht seit April 2018 erstmals eine nicht antibiotische Substanz zur Prävention der Rekurrenz einer CDI bei Erwachsenen mit hohem CDI-Rekurrenzrisiko zur Verfügung. Mit dem Präparat lässt sich die Rekurrenzrate deutlich senken. Bezlotoxumab ist ein humaner, monoklonaler Antikörper, der gegen Toxin B gerichtet ist und dessen Aktivität unterbindet. Toxin B wird beim Auskeimen persistierender oder neu erworbener Clostridium-difficileSporen gebildet. Das neue Medikament wirkt, ohne - im Gegensatz zu Antibiotika - die Zusammensetzung des intestinalen Mikrobioms zu beeinträchtigen. Bezlotoxumab wird während einer antibakteriellen Therapie als intravenöse Einmalinfusion (10 mg/kg Körpergewicht) eingesetzt.

Quelle: www.aerztezeitung.de 